This item was submitted to Loughborough's Research Repository by the author.

Items in Figshare are protected by copyright, with all rights reserved, unless otherwise indicated.

\title{
Pulmonary adaptations to swim and inspiratory muscle training
}

PLEASE CITE THE PUBLISHED VERSION

http://dx.doi.org/10.1007/s00421-008-0759-x

PUBLISHER

(C) Springer-Verlag

VERSION

AM (Accepted Manuscript)

LICENCE

CC BY-NC-ND 4.0

REPOSITORY RECORD

Mickleborough, Timothy D., Joel M. Stager, Ken Chatham, Martin R. Lindley, and Alina A. lonescu. 2019.

"Pulmonary Adaptations to Swim and Inspiratory Muscle Training". figshare. https://hdl.handle.net/2134/11340. 
This item was submitted to Loughborough's Institutional Repository (https://dspace.lboro.ac.uk/) by the author and is made available under the following Creative Commons Licence conditions.

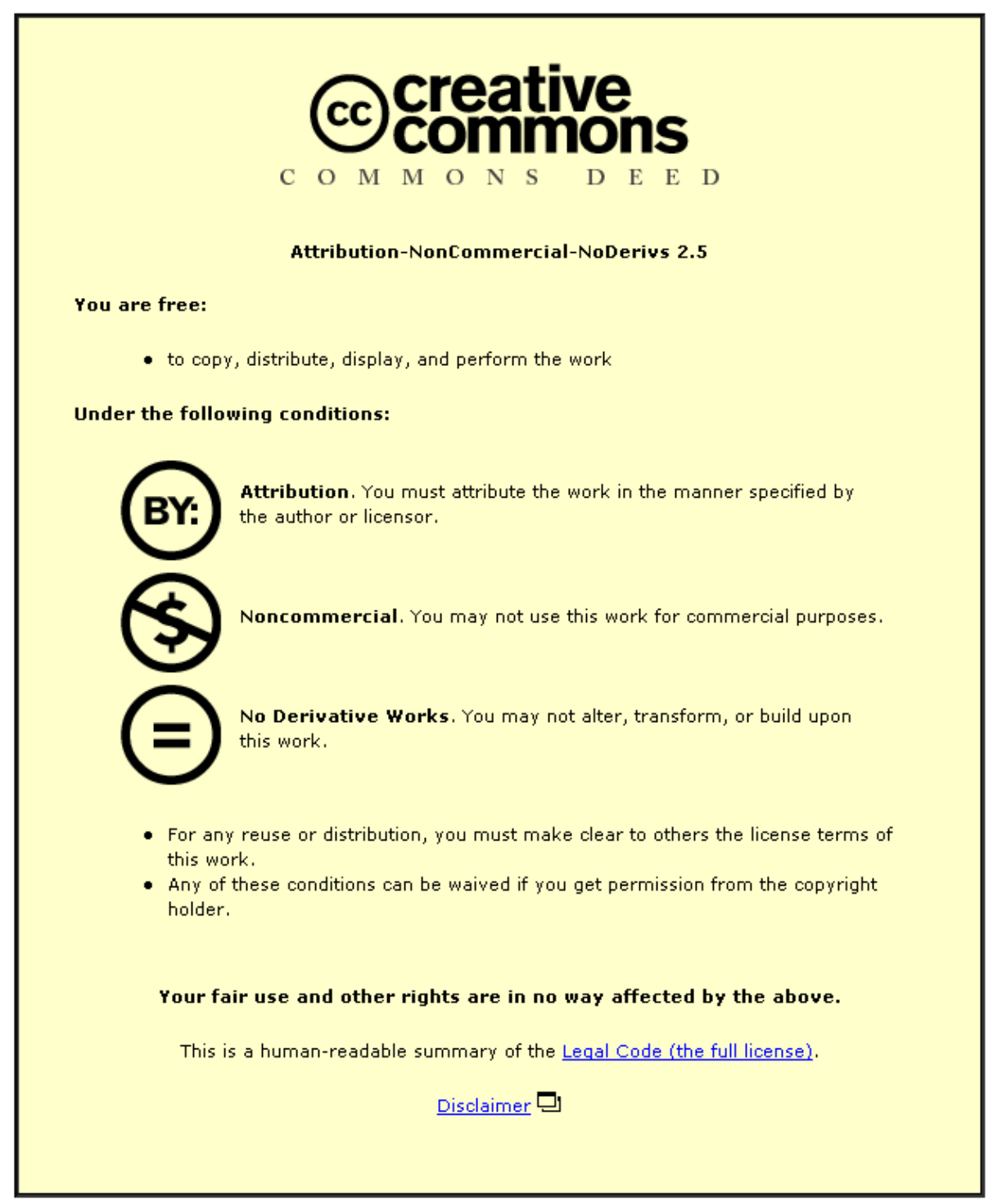

For the full text of this licence, please go to: http://creativecommons.org/licenses/by-nc-nd/2.5/ 


\section{Pulmonary adaptations to swim and inspiratory muscle training}

Timothy D. Mickleborough, Ph.D. ${ }^{1,2,3}$

Joel M. Stager, Ph.D. ${ }^{1,2}$

Ken Chatham, MSCP. ${ }^{5}$

Martin R. Lindley, Ph.D. ${ }^{3,4}$

Alina A. Ionescu, M.D. ${ }^{6}$

${ }^{1}$ Human Performance Laboratory, Department of Kinesiology and ${ }^{2}$ Councilman Center for the Science of Swimming, Indiana University, Bloomington, IN 47405 USA;

${ }^{3}$ School of Sport Science, Physical Education and Recreation, University of Wales Institute, Cardiff, Cyncoed Road, Cardiff, CF23 6XD, United Kingdom;

${ }^{4}$ Department of Human Sciences, Loughborough University, Loughborough, LE11 3TU, United Kingdom;

${ }^{5}$ Physiotherapy Department and ${ }^{6}$ Section of Respiratory Medicine, University of Wales College of Medicine, University Hospital of Wales and Llandough Hospital, NHS Trust, Cardiff, CF64 2XX, United Kingdom.

\section{Correspondence:}

Timothy D. Mickleborough, Ph.D., FACSM

\section{Human Performance Laboratory}

Department of Kinesiology

Indiana University

Bloomington, IN 47405 USA

Tel: (812) 8550753

Fax: (812) 812) 8553193

Email: tmickleb@indiana.edu 


\section{ABSTRACT}

Because the anomalous respiratory characteristics of competitive swimmers have been suggested to be due to inspiratory muscle work, the respiratory muscle and pulmonary function of 30 competitively trained swimmers was assessed at the beginning and end of an intensive 12-week swim training (ST) program. Swimmers $(\mathrm{n}=10)$ combined ST with either inspiratory muscle training (IMT) training (80\% sustained maximal inspiratory pressure (SMIP) with progressively increased work-rest ratios until task failure for 3-d per week (ST + IMT +)) or swim training with sham-IMT (ST + SHAM,-n=10), or acted as controls (swim training only, ST, n=10). Measures of respiratory and pulmonary function were assessed at the beginning and end of the 12 week study period. There were no significant differences $(p>0.05)$ in respiratory and pulmonary function between groups (ST + IMT, IMT + SHAM-IMT and ST) at baseline and at the end of the 12 week study period. However, within all groups significant increases $(\mathrm{p}<0.05)$ were observed in a number of respiratory and pulmonary function variables at the end of the 12 week study, such as maximal inspiratory and expiratory pressure, inspiratory power output, forced vital capacity, forced expiratory and inspiratory volume in 1-sec, total lung capacity and diffusion capacity of the lung. This study has demonstrated that there are no appreciable differences in terms of respiratory changes between elite swimmers undergoing a competitive swim training program and those undergoing respiratory muscle training using the flow-resistive IMT device employed in the present study; as yet, the causal mechanisms involved are undefined.

Keywords: swimming $\bullet$ respiratory $\bullet$ training $\bullet$ pulmonary function 


\section{INTRODUCTION}

Historically, the untrained healthy pulmonary system has been considered to be endowed with sufficient capacity to manage any additional stressors that exercise may impose on it. This position is supported by data indicating that prolonged aerobic training programs cause few morphological and/or functional changes in the healthy adult lung (Dempsey et al. 1977). Studies have shown lung volume to be fairly well predicted based on height and age in individuals engaged in land-based training programs (Kaufmann and Swenson 1981; Raven 1977) and vital capacity (VC) to be unaffected by short-term landbased training (Kollias et al. 1972; Reuschlein et al. 1968; Sinning and Adrian 1968). Thus, for all intents and purposes, neither lung size nor lung function appears to be affected by routine intensive exercise.

However, swimmers appear to be a special group of athletes with lung volumes greater than predicted and with larger VC and total lung capacities (TLC) when compared to controls (Andrew et al. 1972; Cordain et al. 1990; Doherty and Dimitriou 1997; Pherwani et al. 1989; Zinman and Gaultier 1986). As well, enhanced pulmonary diffusion capacity has been reported when swimmers are compared to predicted values (Armour et al. 1993; Miller et al. 1989; Vaccaro et al. 1980) and non-athletes (Yost et al. 1981). Interestingly, Mostyn et al. (Mostyn et al. 1963) found that while collegiate swimmers, long distance runners and non-athletes exhibited no significant deviation from predicted values for pulmonary diffusion capacity, elite swimmers did exhibit significantly higher diffusion capacities when values are compared to predicted norms.

It is unknown at present whether the greater lung volumes observed in swimmers is a result of the swim training itself (Andrew et al. 1972; Clanton et al. 1987; Cordain and Stager 1988), genetic endowment (Barr-Or et al. 1994), increases in respiratory muscle strength (Clanton et al. 1987; Doherty and Dimitriou 1997; Zauner and Benson 1981), changes in the compliance of the chest wall (Clanton et al. 1987; Zauner and Benson 1981), alveolar hyperplasia or expansion (Armour et al. 1993; Zauner and Benson 1981) or the promotion of isotropic lung growth (Courteix et al. 1997). The explanation could 
also include selective factors in which larger lung volumes or enhanced pulmonary function could allow for enhanced swim performance such that as the swim population becomes more elite and lung physiology begins to deviate more from normal values.

Clanton et al. (Clanton et al. 1987) has shown that, while pulmonary function and inspiratory muscle strength and endurance are increased following 12 weeks of swim training in female varsity swimmers, no further changes are noted when additional pressure-threshold inspiratory muscle training (IMT) is combined with swim training. These results conflict with those reported for subjects engaged in IMT but without swim training. Similarly, Wells et al. (Wells et al. 2005) recently demonstrated in adolescent competitive swimmers that 12 weeks of swim training was as effective as swim training plus pressure-threshold respiratory muscle training in eliciting improvements in respiratory muscle strength. These studies seem to suggest that swim training elicits effects upon the pulmonary system similar to, if not greater than, those of isolated ventilatory muscle training. Nevertheless, flow restriction devices are being marketed to swimmers and their coaches and are being widely incorporated into use during swim training bouts as a means to improve swim performance. The merit of these devices is, in general, lacking from the scientific perspective.

Therefore, the purpose of this study was to gain additional insight into respiratory muscle performance and pulmonary function of nationally ranked, physically mature competitive swimmers and the interplay between swim training and flow-resistive IMT. There is very limited data available regarding the adaptive response of the pulmonary system to intensive swim training in elite swimmers training and competing at the highest competitive level. Based upon the results of previous studies, we hypothesized that 12 weeks of swim training would increase strength and endurance of the respiratory muscles and improves lung function and pulmonary diffusion capacity in elite swimmers. We further hypothesised that supplementing the 12 week swim training with IMT would result in no additional enhancement in respiratory muscle performance, lung volume and pulmonary function. 


\section{METHODS}

\section{Subjects}

Thirty competitively trained elite swimmers [15 male and 15 female; mean age 18.2 (SD1.6) years, mean height 171.3 (SD 4.2) cm, mean body mass 68.2 (SD 9.5) kg], all of whom have achieved qualification times standards for either their National Championships, Commonwealth Games, European Games or World Championships, participated in this study. The athletes' swim specialities ranged from 12 sprint distance (50 or $100 \mathrm{~m}$ ), 12 middle distance ( 200 or $400 \mathrm{~m}$ ) to 6 long distance ( 800 to $1500 \mathrm{~m}$ ) events. The study's protocols and procedures were approved by the Local Research Ethics Committee and subjects gave written informed consent to participate in the study. All participants had normal spirometry and had no history of respiratory disease.

\section{Study design and protocol}

Prior to entering the study protocol all subjects underwent a 1 week orientation period for all laboratory-based respiratory muscle and pulmonary function tests in order to remove any potential learning effect (Eastwood et al. 1998; Lindley et al. 2004).

Following the laboratory-based familiarization tests, subjects were randomly assigned (from each gender and swim specialization) to one of three experimental groups: 1) Swim training plus IMT (ST + IMT; n=10); 2) Swim training plus 'sham' IMT ( ST + SHAM-IMT; n=10); and 3) the control group, i.e., swim training only (ST; $\mathrm{n}=10)$. Each group was comprised of 4 sprinters, 4 middle distance and 2 long distance swimmers. Subsequently, all subjects had their respiratory muscle and pulmonary function assessed at baseline over the course of 2 days. On study day 1 respiratory muscle function was assessed; the series of tests conducted on study day 1 comprised an inspiratory muscle strength test followed 7 hours afterwards by a maximal expiratory muscle strength and an inspiratory muscle endurance test. On study day 2 pulmonary function was assessed, which comprised of a spirometry test followed 4 hours 
later by lung volume measurements, after which a diffusion capacity of the lung $\left(\mathrm{DL}_{\mathrm{CO}}\right)$ test was performed 4 hours after the lung volume measurements.

Thereafter, the ST + IMT and ST + SHAM-IMT groups reported to the laboratory 3 times per week (same day and same time of the week) to perform IMT during the 12-week study period. The ST group performed no IMT during the 12 week study period. Following the 12 week study period all subjects again reported to the laboratory to have their pulmonary and respiratory muscle function reassessed in the same manner and order as conducted at baseline.

\section{Spirometry and lung volume measurements}

Spirometry was performed with the subject in the sitting position while breathing room air, with the nose being occluded by a clip. All testing was completed using a calibrated computerized spirometer (Superspiro; Micro Medical; Rochester, Kent, UK), according to American Thoracic Society recommendations (1995b). The pulmonary function technician and spirometer were the same throughout the study. The procedure for all spirometry tests was 1) three normal tidal volume breaths, 2) maximal inhalation, 3) forced maximal exhalation, and 4) maximal inhalation. The best of three consistent trials was recorded. Residual lung volume and TLC were measured using the nitrogen dilution method with a SensorMedics V6200 Autobox (VIASYS Healthcare, Warwick, UK) as previously described (Wilmore et al. 1980).

\section{Diffusion capacity of the lung}

The diffusion capacity of the lung $\left(\mathrm{DL}_{\mathrm{CO}}, \mathrm{ml}^{\cdot} \mathrm{min}^{-1} \mathrm{mmHg}^{-1}\right.$ ) measurements were performed according to the American Thoracic Society standard recommendations for the single-breath carbon monoxide diffusing capacity (transfer factor) (1995a), using a SensorMedics V6200 Autobox (VIASYS Healthcare, Warwick, UK). Each subject wore a nose clip and remained seated throughout the $\mathrm{DL}_{\mathrm{CO}}$ procedure. Subjects exhaled to residual lung volume (RV), inhaled a gas mixture of $20.9 \% \mathrm{O}_{2}, 0.3 \% \mathrm{CO}$, $0.3 \% \mathrm{CH}_{4}$ balanced with $\mathrm{N}_{2}$ to total lung capacity, held their breath for 10 -sec, and then exhaled to RV. 
Between trials 2 min were allowed to permit the washout of gases. $\mathrm{DL}_{\mathrm{CO}}$ measurements were accomplished in duplicate and averaged if within $10 \%$ or $3 \mathrm{ml} \cdot \mathrm{min}^{-1} \cdot \mathrm{mmHg}^{-1}$. A fingertip sample of capillary blood was collected onto a microcuvette and the hemoglobin concentration measured using a Hemocue ${ }^{\circledR}$ B-hemoglobin photometer (Hemocue, Inc., Mission Viejo, CA) in order to correct $\mathrm{DL}_{\mathrm{CO}}$ values (1995a). The transfer coefficient $\left(\mathrm{K}_{\mathrm{CO}}\right)$ was calculated as mean $\mathrm{DL}_{\mathrm{CO}}$ (standard temperature and pressure, dry (STPD) divided by mean alveolar volume (BTPS) (1995a).

\section{Inspiratory muscle training protocol.}

The ST + IMT and ST + SHAM-IMT group performed inspiratory muscle training 3 times per week in the laboratory and under supervision. The device used was the RT2 trainer and associated software (DeVilbiss Sunrise Medical Ltd, Wollaston, UK). The RT2 training device is a pressure manometer with a $2 \mathrm{~mm}$ leak, which utilises an infra-red link to a computer containing the software of the "Test of Incremental Respiratory Endurance" (TIRE) regimen, as previously described (Chatham et al. 1999; Chatham et al. 2004; Enright et al. 2004; Gething et al. 2004a; Gething et al. 2004b). The 2mm leak provides a set resistance to inspiratory flow. The TIRE protocol requires each subject to forcefully exhale to RV (expiration unloaded), followed immediately by each subject breathing in maximally against the resistance ( $2 \mathrm{~mm}$ leak) from RV to total lung capacity (TLC) until task failure. This effort was recorded on a computer screen as sustained maximum inspiratory pressure (SMIP), which is the area under the curve (Figure $2 \mathrm{a}$ and $2 \mathrm{~b}$ ). The best of three SMIP manoeuvres was selected and visually redrawn on the computer screen to a training template set at $80 \%$ (ST + IMT group) or 30\% (ST + SHAM-IMT group) of the peak pressure achieved over the time of inspiration. This fixed the pressure load over the full inspiratory volume range. The $80 \%$ (ST + IMT group) or 30\% (ST + SHAM-IMT group) SMIP training template was presented on a computer screen together with a countdown clock and scores based on the pressures achieved expressed in pressure time units (PTUs). This provided computerized biofeedback to each subject during training, whilst scores were recorded to the computer database and provided further feedback of any training progress. A set training regimen then required 
that the on-screen template was matched or exceeded by participants within a progressively increased work-rest ratio. The initial rest period was 1-min for each of the six resisted inspirations of level A (i.e., 6 inspirations with a 1-min rest period). After level A was successfully completed level B was immediately introduced, which required an additional six resisted inspirations with a 45-sec rest period following each inspiration. At levels $\mathrm{C}$ through $\mathrm{F}$ the rest period between the 6 inspirations was reduced to $30,15,10$ and finally 5-sec respectively. Subjects ceased training if failing to achieve at least $90 \%$ of the on-screen training template, or if they achieved success at the F6 level (a total of 36 inspirations set at the $80 \%$ template). At each IMT session the SMIP was reassessed and the training template for that day set at $80 \%$ (ST + IMT group) or 30\% (ST + SHAM-IMT group) of the new maximal effort. The subjects were

seated and wearing nose clips during the TIRE-IMT protocol. At the conclusion of each IMT session data was stored in the computer for future analysis.

The electronic manometer was calibrated to give conversions from pressure to energy and power. The algorithm used raw data from the manometer, in the form of the vector $\mathrm{p}$, representing the pressure differential measured across the device, sampled at 0.1 second intervals. A leak calibration constant was calculated, from flow rate $(\mathrm{Q})$, as follows:

$$
\mathrm{Q}=3.226 \times 10^{-6} \times \sqrt{p}
$$

where pressure $(p)$ was expressed in N. $\mathrm{m}^{2}$ and Q in $\mathrm{m}^{3} \cdot \mathrm{s}^{-1}$. Power (P; in watts) was then calculated as follows:

$$
\mathrm{P}=p \times \mathrm{Q}
$$

The work per breath was derived from the power curve and expressed in Joules (Chatham et al. 2004).

\section{Inspiratory muscle strength}

Inspiratory muscle strength was measured in all subjects (ST + IMT, ST + SHAM-IMT and ST groups) as the maximum negative inspiratory pressure generated at RV and sustained during a maximal inspiration using the RT2 pressure manometer. MIP and SMIP were measured by asking subjects to 
maximally inhale against the set resistance from RV to TLC and were again recorded on the computer screen. This was recorded as an indication of the work performed at each maximal breath, as the inspiratory muscles contracted throughout their full range. The inspiratory time of contraction $\left(\mathrm{T}_{\text {cont }}\right)$ during the SMIP manoeuvre was also recorded.

\section{Inspiratory muscle endurance}

Inspiratory muscle endurance in all subjects (ST + IMT, ST + SHAM-IMT and ST groups) was determined by computing the total accumulated SMIP's ( $\sum$ SMIP or $\sum$ ptu's) generated for each level successfully completed (i.e., A through F) recorded by the RT2 software during a TIRE-IMT session and conducted at the beginning and end of the 12 week study period. As an additional measure of inspiratory muscle endurance, all subjects were asked to match a 75\% SMIP target presented every ten seconds via the countdown clock, and the time to failure $\left(\mathrm{T}_{\text {lim }}\right.$ ) (i.e., unable to match at least $90 \%$ of the target template) was recorded before and after the 12 week study period.

\section{Maximal expiratory muscle strength}

Maximal expiratory muscle strength was assessed by measuring static expiratory mouth pressures using a hand-held respiratory pressure meter (Micro Direct, Inc., Lewiston, ME). Since, these respiratory pressures vary with the point in lung volume that they are performed at, MEP was measured at total lung capacity (2002). The maximal value of 3 manoeuvres that did not vary by less than $10 \%$ was recorded (2002). At least a minute between attempts was allowed to avoid any effect from respiratory muscle fatigue.

\section{Swim training}

All athletes were engaged in a similar swim training program specifically designed to enhance competitive swim performance, and followed the training program set by their coaches. The study was 
started at the beginning of the base training period [i.e., after detraining from the previous swimming year (transition phase)]. During the course of the 12 week study all subjects trained between 10 to 12 swim sessions per week, averaging 40-60 km of swimming per week under coaching supervision, in conjunction with 3 dry land strength training sessions per week. The sprinters, middle distance and long distance swimmers did slightly different training in terms of distance and intensity. For example, the sprinters will typically swim less volume but higher intensity in any given session than the middle and long distance swimmers. Swim training intensity was regularly assessed using the tests of Lavoie et al (Lavoie et al. 1985). The IMT protocol was initiated at the beginning of the swim base training phase. None of the swimmers suffered any major injury during the study that prevented them from training. All participants were required to record training volume, frequency and intensity in a diary during the study period. In addition, a questionnaire was administered apriori to determine past and recent swim training history.

\section{Statistical analysis}

Data were analyzed using SPSS version 15.0 statistical software (SPSS Inc., Chicago, USA). All data were assessed for normality using the Kolmogorov-Smirnov test, and Levene's test was used to test for homogeneity of variance between groups. The respiratory muscle and pulmonary function data were analyzed using paired (within group) and unpaired (between groups) t-tests. Data are expressed as mean and their 95\% confidence interval (CI). Statistical significance was accepted if $\mathrm{p}<0.05$.

\section{RESULTS}

\section{Subjects}

There were no significant differences $(\mathrm{p}>0.05)$ in age, height, arm length and body mass between groups, and no significant differences $(\mathrm{p}>0.05)$ were observed in these anthropometric variables over the course of the study period. In addition, no significant differences ( $p>0.05)$ were noted between the three groups for swim training frequency, volume or intensity. 


\section{Pulmonary function}

There was no significant difference ( $\mathrm{p}>0.05)$ between groups (ST + IMT; ST + SHAM-IMT; ST) at baseline or at the end of the 12 week study period for forced vital capacity (FVC), forced expiratory volume in 1-second $\left(\mathrm{FEV}_{1}\right)$, forced inspiratory volume in 1-second $\left(\mathrm{FIV}_{1}\right)$, residual volume (RV), TLC, $\left.\mathrm{DL}_{\mathrm{CO}}\right)$ or $\mathrm{K}_{\mathrm{CO}}$ (Table 1, 2 and 3). However, within all groups significant changes $(\mathrm{p}<0.05)$ were observed in a number of pulmonary function variables at the completion of the 12 week study period (Figure 1a). For the ST + IMT group (Table 1 and Figure 1a) there was a significant improvement $(\mathrm{p}<0.05)$ of $11.6 \pm$ $5.6 \%, 13.3 \pm 6.1 \%, 7.3 \pm 4.8 \%, 7.4 \pm 4.0 \%$ and $13.1 \pm 5.5 \%$ in FVC, $\mathrm{FEV}_{1}, \mathrm{FIV}_{1}, \mathrm{TLC}$ and $\mathrm{DL}_{\mathrm{CO}}$ respectively at the end of 12 week conditioning period compared to baseline. At the completion of the 12 week conditioning period the ST + SHAM-IMT group (Table 2 and Figure 1a) demonstrated significant improvements $(\mathrm{p}<0.05)$ in $\mathrm{FVC}, \mathrm{FEV}_{1}, \mathrm{FIV}_{1}$, TLC and $\mathrm{DL}_{\mathrm{CO}}$ of $10.9 \pm 4.9 \%, 12.7 \pm 5.6 \%, 6.5 \pm 5.1 \%, 6.6$ $\pm 3.9 \%$ and $11.2 \pm 4.7 \%$ respectively compared to baseline. The ST group (Table 3 and Figure 1a) exhibited significant pulmonary function improvements $(\mathrm{p}<0,05)$ following the 12 week study period in $\mathrm{FVC}, \mathrm{FEV}_{1}, \mathrm{FIV}_{1}$, TLC and $\mathrm{DL}_{\mathrm{CO}}$ of $11.2 \pm 5.2 \%, 11.1 \pm 6.5 \%, 7.5 \pm 5.4 \%, 6.4 \pm 4.6 \%$ and $8.9 \% \pm 4.4 \%$ respectively. However, there was no significant difference $(\mathrm{p}<0.05)$ between groups $(\mathrm{ST}+\mathrm{IMT}$; ST + SHAM-IMT; ST) at the end of the 12 week condition period in the $\%$ improvement in all measures of pulmonary function.

\section{Respiratory muscle function}

No significant difference $(\mathrm{p}>0.05)$ was observed in respiratory muscle function between groups (ST + IMT; ST + SHAM-IMT; ST) at baseline or at the end of the 12 week study period (Table 1, 2 and 3; Figure $2 \mathrm{a}$ and $2 \mathrm{~b}$ ). However, all measured respiratory muscle function variables within all groups significantly increased $(\mathrm{p}<0.05)$ at the end of the 12 week study period (Table 1, 2 and 3; Figure 1b). At the end of the 12 week study period there was a significant increase $(\mathrm{p}<0.05)$ in MIP and inspiratory 
muscle PO within all groups compared to baseline [ST + IMT group: MIP, $46.5 \pm 8.3 \%$ and SMIP, (joules) $49.6 \pm 6.2 \%$ respectively; ST + SHAM-IMT group: MIP, $35.4 \pm 6.7 \%$ and SMIP (joules) $47.9 \pm$ $5.7 \%$ respectively; ST group: MIP, $31.2 \pm 5.6 \%$ and SMIP (joules) $47.2 \pm 6.1 \%$ respectively)]. In addition, inspiratory muscle endurance (ST + IMT group: $\mathrm{T}_{\text {lim }}, 17.5 \pm 2.6 \%$; ST + SHAM-IMT group: Tlim, $10.7 \pm 2.3 \%$; ST group: Tlim, $14.1 \pm 2.4 \%)$ and expiratory muscle strength (ST + IMT group: MEP, $40.1 \pm 6.4 \% ;$ ST + SHAM-IMT group: MEP, $39.3 \pm 4.8 \% ;$ ST group: MEP, $45.1 \pm 5.3 \%)$ significantly increased $(\mathrm{p}<0.05)$ at the end of the 12 week study period. However, there was no significant difference $(\mathrm{p}<0.05)$ between groups $(\mathrm{ST}+\mathrm{IMT} ; \mathrm{ST}+\mathrm{SHAM-IMT} ; \mathrm{ST})$ at the end of the 12 week condition period in the \% improvement in all measures of respiratory muscle function.

\section{DISCUSSION}

The main purpose of the present study was to further evaluate the combined effect of a 12 week swim training and an IMT program on measures of respiratory muscle and pulmonary function in elite swimmers. The results of the present study have demonstrated that a rigorous 12 week competitive swim training program improves pulmonary function and increases respiratory muscle strength and endurance in elite swimmers to the same to the same extent as a 12 week combined IMT and competitive swim training program. Or, stated in the opposite direction, IMT in addition to a rigorous swim training program does not result in additional pulmonary or respiratory muscle adaptations beyond that of swim training alone.

\section{Pulmonary function}

Our results support the work of Clanton et al. (Clanton et al. 1987) who similarly observed increases in pulmonary and respiratory muscle function after 12 weeks of swim training in 16 female collegiate swimmers, but found no additional adaptations in these measures when pressure-threshold IMT was added to the training regime. Wells et al. (Wells et al. 2005) also report that swim training plus sham concurrent inspiratory and expiratory muscle pressure-threshold training (CRMT) is as effective as swim 
training plus CRMT in eliciting improvements in inspiratory muscle strength. They report, however, statistical improvements in several measures of pulmonary function $\left(\mathrm{FEV}_{1}\right.$ and $\left.\mathrm{FIV}_{\mathrm{I}}\right)$ that were only observed in the swim training plus CRMT and not the swim training plus sham-CMRT group.

Consistent with this, all of our subjects demonstrated increases in $\mathrm{FIV}_{1}$, which we hypothesize may be a direct result of an increase in the velocity of shortening of the inspiratory muscles as a consequence of enhanced inspiratory muscle strength. The functional outcome of this is to make it possible to achieve faster maximum inspirations (Clanton et al. 1987). This may be particularly important for elite swimmers since this would allow them to increase the amount of air they can inhale in the limited time their face is out the water. In contrast to many athletes, where expiratory time is perhaps limiting to maximum minute ventilation during exercise, a swimmer's minute ventilation, because of the obligatory nature of the timing of the inspiratory phase of the breathing cycle which coincides with arm movements, may be limited by inspiratory time.

The present study confirms previous findings that swim training augments static and dynamic lung volumes (Andrew et al. 1972; Clanton et al. 1987; Courteix et al. 1997). It has been suggested that the repeated expansion of the of the lung to TLC, which occurs during moderate- to high-intensity swim training is the stimulus for the enhanced VC and TLC observed in swimmers (Cordain and Stager 1988). An increase in inspiratory force production near TLC in swimmers may occur as a consequence of large tidal volumes being achieved during swimming, conditioning of the accessory neck and chest wall muscles, or a shift in the length of the inspiratory muscles, such that larger forces could be generated at shorter muscle lengths (Clanton et al. 1987). Alternately, the observed increase in TLC may be the result of a change in lung compliance or by lung growth (alveolar hyperplasia/hypertrophy), which may shift the volume-pressure curve for the lung and chest wall, making it easier to expand the thorax with a lesser amount of inspiratory muscle force (Clanton et al. 1987). Fanta et al. (Fanta et al. 1983) have shown significant increases in VC occur only if inspiratory muscle exercises are performed near TLC, providing evidence that the inspiratory muscles can be trained for increased maximal shortening. Therefore, it is 
feasible that the increase in $\mathrm{FVC}$ and $\mathrm{FEV}_{1}$ over the course of the 12 week swim conditioning period in the present study may occur concomitant with, and be attributed to, the increase in TLC.

Our findings are in agreement with others that elite swimmers exhibit elevated $\mathrm{DL}_{\mathrm{CO}}$ values, greater than predicted (Armour et al. 1993; Magel and Andersen 1969; Miller et al. 1989; Mostyn et al. 1963; Vaccaro et al. 1977) and greater than non-athletes (Andrew et al. 1972; Yost et al. 1981), even when corrected for body size and total lung capacity $\left(\mathrm{K}_{\mathrm{CO}}\right)$ (Magel and Andersen 1969; Mostyn et al. 1963; Yost et al. 1981). It has been suggested that elite swimmers have a high $\mathrm{DL}_{\mathrm{CO}}$, as they must be able to transfer large amounts of oxygen across the alveolar-capillary membrane when the alveolar oxygen tension $\left(\mathrm{PaO}_{2}\right)$ falls to low levels (Mostyn et al. 1963), and that the enlarged $\mathrm{DL}_{\mathrm{CO}}$ is a consequence of an increased pulmonary capillary blood volume (Miller et al. 1989). It is unclear at present whether an augmented $\mathrm{DL}_{\mathrm{CO}}$ observed in elite swimmers is due to long term swim training or is an innate characteristic.

\section{Respiratory muscle function}

Inspiratory muscle strength and endurance, expiratory muscle strength, and inspiratory muscle power output (PO) significantly increased in all test groups, but were not significantly different between groups, at the end of the 12 week conditioning period. The present study is in agreement with prior studies (Clanton et al. 1987; Wells et al. 2005) showing inspiratory muscle strength increases as a result of competitive swim training. In addition, the work per breath derived from the SMIP power curve (joules) increased following the 12 week swim conditioning period. There is very little information on the effect of inspiratory muscle conditioning on inspiratory flow, and thus on inspiratory muscle PO. As with other skeletal muscles, it has been shown that the inspiratory muscles can be trained to increase their capacity to generate force (pressure) or muscle shortening velocity (flow) (Leith and Bradley 1976;

Tzelepis et al. 1999; Tzelepis et al. 1994). During swimming (flow-targeted type training) a higher tidal volume may cause encroachment on end-inspiratory reserve volume, which requires the inspiratory muscles to shorten at a high velocity during the rapid inspiration as the face leaves the water (Cordain and 
Stager 1988; Fanta et al. 1983). Tzelepis et al. (Tzelepis et al. 1999) found that flow-targeted training diminished the time to reach the peak oesophageal pressure by about 25\%, when performing IMT. Thus, during swimming, it is feasible that the enhanced airway resistive load due to high flow rates during inspiration and expiration (Courteix et al. 1997; Kohl et al. 1997) increases strength and shortening velocity of the inspiratory muscles, and thus, in PO. Since myosin heavy-chain composition is one of the determinants of shortening velocity (Linari et al. 2004), a possible mechanism underlying the improvement in PO with training may be the changes in myosin heavy-chain subtypes shown in the diaphragm of experimental animals (Bottinelli et al. 1991) and human vastus lateralis muscle fibers (Linari et al. 2004), and/or hypertrophy of predominantly type IIa muscle fibers (Bisschop et al. 1997; Rollier et al. 1998). However, further research is needed to establish the role of such changes in the increase in PO due to inspiratory muscle conditioning.

The present study is in agreement with prior studies that have reported improvements in inspiratory muscle endurance in swimmers (Clanton et al. 1987; Martin and Stager 1981). Martin and Stager (Martin and Stager 1981) documented similar respiratory endurance between endurance-trained athletes (swimmers/runners) and non-athletes during short-term tests, but increased respiratory endurance in the athletes on longer term tests (Martin and Stager 1981). This increased respiratory muscle endurance is consistent with data from animal experiments that demonstrate increased oxidative metabolism activity in the diaphragm following various types of endurance training (Powers et al. 1992; Powers et al. 1990). That IMT results in additional benefits or adaptations when added to a rigorous swim training program cannot be supported from analysis of the present data.

It is well known that females exhibit a number of anatomic and physiologic characteristics that distinguish their responses to exercise from those of men. These differences may have an effect on the integrated ventilatory response, respiratory muscle work, and in pulmonary gas exchange during exercise (Harms 2006). In the present study, no sex differences in pulmonary and respiratory muscle function were detected between sexes at the end of the 12 week study period. However, the study was not statistically 
powered to detect sex differences in these measures at the end of the study period, and was only powered to detect differences between groups, with males and females combined in each group $(n=10)$.

\section{Influence of IMT on pulmonary and respiratory muscle function}

What is a likely explanation as to why combining IMT with swim training conferred no additional improvement in respiratory muscle and pulmonary function measures compared to the swim training program alone? There are a number of feasible explanations. Firstly, it is possible that the IMT device and the protocol employed resulted in insignificant and sub-optimal adaptations to the respiratory musculature. However, this seems doubtful since the RT2 inspiratory muscle trainer used in the present study, which utilizes inspiratory flow resistive loading and the TIRE protocol, has previously been shown to improve both the strength and endurance of the inspiratory muscles. Improved cycling capacity in healthy subjects (Gething et al. 2003; Gething et al. 2004b) has been reported. Increased strength and endurance of the inspiratory muscles, enhanced lung volume (VC), increased diaphragm thickness and greater exercise capacity in healthy subjects (Enright et al. 2006) has also been shown with similar equipment and protocols. Enhanced inspiratory muscle strength and endurance, and exercise capacity in recreational runners (Mickleborough et al. 2003) has been reported, and improved lung function and exercise capacity in patients with cystic fibrosis has been described (Enright et al. 2004). It therefore seems unlikely that either equipment or protocol induce ventilatory stimuli that are of insufficient magnitude to initiate an adaptive response given those previously reported in the literature.

Secondly, swim training places unique stressors upon the pulmonary system. A swimmer must

coordinate both tidal volume and breathing frequency with stroke mechanics, which results in inspiration becoming a rapid, forced manoeuvre, thus respiratory frequency tends to be lower and a higher tidal volume compared to spontaneously breathing exercises (Rodriguez 2000). Novice swimmers tend to manipulate arm movements such that breathing is not limited. In contrast, more experienced swimmers tend to manipulate breathing such that propulsive movements are not limited by breathing. In order to do 
so, the swimmer is forced into an "obligatory controlled frequency breathing" pattern. Thus, the respiratory pattern becomes one of a "gasp" inhalation and a relatively prolonged exhalation.

The resultant rapid, 'forced' nature of inspiration during swimming, and the additional increased hydrostatic pressure upon the thorax, may result in pushing the chest wall inwards when the inspiratory muscles are relaxed (Cordain and Stager 1988). As hydrostatic pressure counters inspiratory muscle force, it is clear that the work performed by the inspiratory muscles must substantially increase in attempt to overcome these factors (Cordain and Stager 1988). Therefore, it is possible that the increased hydrostatic pressure during submersion, and high flow rates experienced during inspiration, may increase the airway resistive load and provide a substantial conditioning stimulus to the inspiratory muscles during swimming (Courteix et al. 1997). However, this may not be the case for all strokes, backstroke might be the exception. It has been suggested that the degree of change in inspiratory muscle strength may be more noticeable in child swimmers compared to adolescents and young adult swimmers, particularly when force (estimated as the product of minimal pressures and thorax surface area) is expressed to total lung volume (Zinman and Gaultier 1986)..

It might also be suggested that the IMT "load" might be insignificant when included in the common program that competitive swimmers are engaged. It is not uncommon for mature, highly competitive swimmers to train for four or more hours per day, 6 days a week. The effects of the IMT protocol which requires approximately 30 minutes per session simply may be overwhelmed by the swim training effects superimposed over the top of it or unlikely but possible, swim training might simply inhibit the expected responses to IMT. A mechanism accounting for this "inhibitory" or "masking" effect is difficult to describe.

In any event, both inspiration and expiration, which cannot occur ad libitum during swimming, may represent a substantial resistive load upon the respiratory musculature, such that combining IMT to a competitive swim training program, results in no further improvement in pulmonary and respiratory muscle function in elite swimmers. It is conceivable that the swim training program per se could stimulate a significant change in the force-length characteristics of the inspiratory muscles by significantly 
increasing their maximum shortening velocity, such that any additional conditioning stimulus (e.g., IMT) may have negligible effects on inspiratory muscle strength and endurance.

Since the subjects in the present study were considered to be physically mature (mean age; $18.2 \pm$ 1.6 years) it is possible, but unlikely, that growth during the study period may have influenced the observed changes in pulmonary and respiratory muscle function. However, no significant changes in anthropometric variables were observed during the course of the 12 week conditioning period, and so were unlikely to have been responsible for the observed changes in pulmonary and respiratory muscle function. According to the predictive equations established by Schoenberg et al. (Schoenberg et al. 1978) the increase in FVC that occurred during the 12 week study period markedly exceeded values that could be attributed to normal lung growth and development during the young adult years (Schoenberg et al. 1978), which suggests that the augmented lung volumes documented in the elite swimmers in the present study may, in part, be due to the swim training stimulus and not the consequence of being endowed with inherently large lung volumes (Clanton et al. 1987; Courteix et al. 1997). What might be suggested from these data, however, is that swim training and IMT appear to result in similar adaptive stressors.

\section{Implications for swim performance}

The present study was not designed to address the question of whether adding IMT to a competitive swim training program would result in improved swimming performance. While several studies have shown that IMT can improve exercise performance in a variety of sports (McConnell and Romer 2004), only two studies to date have assessed the impact of IMT on swim performance. Wells and coworkers (Wells et al. 2005) have shown that 12 weeks of CRMT can increase critical swimming speed in female, but not male, adolescent competitive swimmers, whereas Wylegala et al. (Wylegala et al. 2007) recently demonstrated that 4 weeks of respiratory muscle training improved swimming endurance in scuba divers. Interestingly, research on competitive Masters Swimmers has shown that a single $200 \mathrm{~m}$ freestyle swim corresponding to $90-95 \%$ of race pace was sufficient to induce inspiratory muscle fatigue (IMF) (Lomax and McConnell 2003). Whether this IMF developed during swimming (Lomax and 
McConnell 2003) impairs swim performance has not been studied. In contrast, slight or no IMF has been shown to occur after the swim phase of a triathlon (Hill et al. 1991). Further studies are clearly needed to evaluate the effect of IMT on swim performance and to clarify the issue of whether swimming exercise induces IMF in competitive swimmers.

\section{Conclusion}

This study has demonstrated that there are no appreciable differences in terms of respiratory changes between elite swimmers undergoing a competitive swim training program and those undergoing flow-resistive IMT; as yet, the causal mechanisms involved are undefined. Further, these results do not support the use of commercially available flow-restrictive devices, which are similar in design to the device used in the present study, that are currently being marketed to swimmers and their coaches as a means to improve swim performance. Our data would suggest that little is to be gained, using the flowresistive IMT device employed in the present study, in terms of pulmonary function beyond that which occurs in response to the swim training itself. Additional studies are needed which assess the efficacy of other respiratory muscle training devices (e.g. pressure-threshold loading and voluntary isocapnic hyperpnea) on pulmonary function and swim performance. 


\section{REFERENCES}

American Thoracic Society (1995a) Single-breath carbon monoxide diffusing capacity (transfer factor).

Recommendations for a standard technique-- 1995 update. Am J Respir Crit Care Med 152:218598

American Thoracic Society (1995b) Standardization of spirometry--1994 update. Am J Respir Crit Care Med 152:1107-1136

Andrew GM, Becklake MR, Guleria JS, Bates DV (1972) Heart and lung functions in swimmers and nonathletes during growth. J Appl Physiol 32:245-51

Armour J, Donnelly PM, Bye PT (1993) The large lungs of elite swimmers: an increased alveolar number? Eur Respir J 6:237-47.

ATS/ERS Statement on respiratory muscle testing (2002) Am J Respir Crit Care Med 166:518-624

Barr-Or O, Unithan V, Illescas C (1994) Physiologic considerations in age group swimming. Med Sport Sci 39:199-205

Bisschop A, Gayan-Ramirez G, Rollier H, Gosselink R, Dom R, de Bock V, Decramer M (1997) Intermittent inspiratory muscle training induces fiber hypertrophy in rat diaphragm. Am J Respir Crit Care Med 155:1583-9

Bottinelli R, Schiaffino S, Reggiani C (1991) Force-velocity relations and heavy chain isoform compositions of skinned fibers from rat skeletal muscle. J Physiol 437:655-672

Chatham K, Baldwin J, Griffiths H, Summers L, Enright S (1999) Inspiratory muscle training improves shuttle run performance in healthy subjects. Physio J 85:676-683

Chatham K, Ionescu AA, Nixon LS, Shale DJ (2004) A short-term comparsion of two methods of sputum expectoration in cystic fibrosis. Eur Respir J 23:435-439 
Clanton TL, Dixon GF, Drake J, Gadek JE (1987) Effects of swim training on lung volumes and inspiratory muscle conditioning. J Appl Physiol 62:39-46

Cordain L, Stager J (1988) Pulmonary structure and function in swimmers. Sports Med 6:271-8

Cordain L, Tucker A, Moon D (1990) Lung volumes and maximal respiratory pressures in collegiate swimmers and runners. Res Quart Exerc Sport 61:70-74

Courteix D, Obert P, Lecoq AM, Guenon P, Koch G (1997) Effect of intensive swimming training on lung volumes, airway resistance and on the maximal expiratory flow-volume relationship in prepubertal girls. Eur J Appl Physiol Occup Physiol 76:264-9

Dempsey JA, Gledhill N, Reddan WG, Forster HV, Hanson PG, Claremont AD (1977) Pulmonary adaptation to exercise: effects of exercise type and duration, chronic hypoxia and physical training. Ann N Y Acad Sci 301:243-61

Doherty M, Dimitriou L (1997) Comparison of lung volume in Greek swimmers, land based athletes, and sedentary controls using allometric scaling. Br J Sports Med 31:337-41

Eastwood PR, Hillman DR, Morton AR, Finucane KE (1998) The effects of learning on the ventilatory responses to inspiratory threshold loading. Am J Respir Crit Care Med 158:1190-6.

Enright S, Chatham K, Ionescu AA, Unnithan VB, Shale DJ (2004) Inspiratory muscle training improves lung function and exercise capacity in adults with cystic fibrosis. Chest 126:405-11

Enright SJ, Unnithan VB, Heward C, Withnall L, Davies DH (2006) Effect of high-intensity inspiratory muscle training on lung volumes, diaphragm thickness, and exercise capacity in subjects who are healthy. Phys Ther 86:345-54

Fanta CH, Leith DE, Brown R (1983) Maximal shortening of inspiratory muscles: effect of training. J Appl Physiol 54:1618-23

Gething A, Williams M, Passfield L, Davies B (2003) A comparison of maximal and submaximal inspiratory muscle training protocols. J Sports Sci 21:322-323 [Abstract]

Gething AD, Passfield L, Davies B (2004a) The effects of different inspiratory muscle training intensities on exercising heart rate and perceived exertion. Eur J Appl Physiol 92:50-5 
Gething AD, Williams M, Davies B (2004b) Inspiratory resistive loading improves cycling capacity: a placebo controlled trial. Br J Sports Med 38:730-736

Harms CA (2006) Does gender affect pulmonary function and exercise capacity? Respir Physiol Neurobiol 151:124-31

Hill NS, Jacoby C, Farber HW (1991) Effect of an endurance triathlon on pulmonary function. Med Sci Sports Exerc 23:1260-4

Kaufmann DA, Swenson EW (1981) Pulmonary changes during marathon training: a longitudinal study. Respiration 41:217-23

Kohl J, Koller EA, Brandenberger M, Cardenas M, Boutellier U (1997) Effect of exercise-induced hyperventilation on airway resistance and cycling endurance. Eur J Appl Physiol Occup Physiol $75: 305-11$

Kollias J, Boileau RA, Bartlett HL, Buskirk ER (1972) Pulmonary function and physical conditioning in lean and obese subjects. Arch Environ Health 25:146-150

Lavoie J-M, Leger LA, Leone M, Provencher PJ (1985) A maximal multistage swim test to determine the functional and maximal aerobic power of competitive swimmers. J Swim Res 1:17-22

Leith DE, Bradley M (1976) Ventilatory muscle strength and endurance training. J Appl Physiol 41:50816

Linari M, Bottinelli R, Pellegrino MA, Reconditi M, Reggiani C, Lombardi V (2004) The mechanism of the force response to stretch in human skinned muscle fibres with different myosin isoforms. $\mathbf{J}$ Physiol (Lond) 554:335-352

Lindley MR, Mickleborough TD, Thorniley CS, Bown RM (2004) Effect of learning and reliability on measures of inspiratory muscle function using a computerised resistive pressure manometer. Am J Respir Crit Care Med 169:A442 [Abstract]

Lomax ME, McConnell AK (2003) Inspiratory muscle fatigue in swimmers after a single $200 \mathrm{~m}$ swim. J Sports Sci 21:659-64 
Magel JR, Andersen KL (1969) Pulmonary diffusing capacity and cardiac output in young trained Norwegian swimmers and untrained subjects. Medicine and Science in Sports 1:131-139

Martin BJ, Stager JM (1981) Ventilatory endurance in athletes and non-athletes. Med Sci Sports Exerc 13:21-6

McConnell AK, Romer LM (2004) Respiratory muscle training in healthy humans: resolving the controversy. Int J Sports Med 25:284-93

Mickleborough TD, Nichols T, Chatham K, Lindley MR, Ionescu AA, Shale DJ (2003) The effect of high and low-intensity inspiratory muscle training on the physiological response to exercise in recreational runners. Am J Respir Crit Care Med 167:A541 [Abstract]

Miller RL, Robison E, McCloskey JB, Picken J (1989) Pulmonary diffusing capacity as a predictor of performance in competitive swimming. J Sports Med Phys Fitness 29:91-6

Mostyn EM, Helle S, Gee JBL, Bentivoglio LG, Bates DV (1963) Pulmonary diffusion capacity of athletes. J Appl Physiol 18:687-695

Pherwani AV, Desai AG, Solepure AB (1989) A study of pulmonary function of competitive swimmers. Indian J Physiol Pharmacol 33:228-32

Powers SK, Criswell D, Lieu F-K, Dodd S, Silverman H (1992) Diaphragmatic fiber type specific adaptation to endurance exercise. Respiration Physiology 89:195-207

Powers SK, Lawler J, Criswell D, Dodd S, Grinton S, Bagby G, Silverman H (1990) Endurance-traininginduced cellular adaptations in respiratory muscles. J Appl Physiol 68:2114-8

Raven PB (1977) Pulmonary function of elite distance runners. Ann N Y Acad Sci 301:371-81

Reuschlein PS, Reddan WG, Burpee J, Gee JB, Rankin J (1968) Effect of physical training on the pulmonary diffusing capacity during submaximal work. J Appl Physiol 24:152-8

Rodriguez FA (2000) Maximal oxygen uptake and cardiorespiratory response to maximal 400-m free swimming, running and cycling tests in competitive swimmers. J Sports Med Phys Fitness 40:8795 
Rollier H, Bisschop A, Gayan-Ramirez G, Gosselink R, Decramer M (1998) Low load inspiratory muscle training increases diaphragmatic fiber dimensions in rats. Am J Respir Crit Care Med 157:833-9

Schoenberg JB, Beck GJ, Bouhuys A (1978) Growth and decay of pulmonary function in healthy blacks and whites. Respir Physiol 33:367-93

Sinning WE, Adrian MJ (1968) Cardiorespiratory changes in college women due to a season of competitive basketball. J Appl Physiol 25:720-4

Tzelepis GE, Kasas V, McCool FD (1999) Inspiratory muscle adaptations following pressure or flow training in humans. Eur J Appl Physiol Occup Physiol 79:467-71.

Tzelepis GE, Vega DL, Cohen ME, Fulambarker AM, Patel KK, McCool FD (1994) Pressure-flow specificity of inspiratory muscle training. J Appl Physiol 77:795-801.

Vaccaro P, Clarke DH, Morris AF (1980) Physiological characteristics of young well-trained swimmers. Eur J Appl Physiol Occup Physiol 44:61-6

Vaccaro P, Zauner CW, Updyke WF (1977) Resting and exercise respiratory function in well trained child swimmers. J Sports Med Phys Fitness 17:297-306

Wells GD, Plyley M, Thomas S, Goodman L, Duffin J (2005) Effects of concurrent inspiratory and expiratory muscle training on respiratory and exercise performance in competitive swimmers. Eur J Appl Physiol 94:527-40

Wilmore JH, Vodak PA, Parr RB, Girandola RN, Billing JE (1980) Further simplification of a method for determination of residual lung volume. Med Sci Sports Exerc 12:216-8

Wylegala JA, Pendergast DR, Gosselin LE, Warkander DE, Lundgren CE (2007) Respiratory muscle training improves swimming endurance in divers. Eur J Appl Physiol 99:393-404

Yost LJ, Zauner CW, Jaeger MJ (1981) Pulmonary diffusing capacity and physical working capacity in swimmers and non-swimmers during growth. Respiration 42:8-14

Zauner CW, Benson NY (1981) Physiological alterations in young swimmers during three years of intensive training. J Sports Med 21:179-185 
Zinman R, Gaultier C (1986) Maximal static pressures and lung volumes in young female swimmers. Respir Physiol 64:229-39

\section{FIGURE LEGENDS}

Figure 1a. The mean $( \pm S D)$ percent change from baseline (prior to 12 week study period) for measures of pulmonary function. The * indicates a significant difference $(\mathrm{p}<0.05)$ compared to baseline. ST + IMT, swim training plus inspiratory muscle training; ST + SHAM-IMT, swim training plus sham-inspiratory muscle training; ST, swim training.

Figure 1b. The mean $( \pm \mathrm{SD})$ percent increase from baseline (prior to 12 week study period) for measures of respiratory muscle function. The * indicates a significant difference $(\mathrm{p}<0.05)$ compared to baseline. ST + IMT, swim training plus inspiratory muscle training; ST + SHAM-IMT, swim training plus shaminspiratory muscle training; ST, swim training.

Figure 2a. Tests of incremental respiratory endurance (TIRE) templates prior to the 12 week study period (baseline). MIP, maximal inspiratory pressure; SMIP, sustained maximal inspiratory pressure (area under the curve); ST + IMT, swim training plus inspiratory muscle training; ST + SHAM-IMT, swim training plus sham-inspiratory muscle training; ST, swim training.

Figure 2b. Tests of incremental respiratory endurance (TIRE) templates at the end of the 12 week study period. MIP, maximal inspiratory pressure; SMIP, sustained maximal inspiratory pressure (area under the curve); ST + IMT, swim training plus inspiratory muscle training; ST + SHAM-IMT, swim training plus sham-inspiratory muscle training; ST, swim training. 
Table 1. Respiratory muscle and pulmonary function values for swim training (ST) and inspiratory muscle training (IMT) group

\begin{tabular}{|c|c|c|c|c|c|c|}
\hline & Pre & Post & Diff & $P$-value & Lower 95\% CI & Upper $95 \% \mathrm{CI}$ \\
\hline \multicolumn{7}{|l|}{ Respiratory Muscle Function } \\
\hline $\mathrm{MIP}\left(\mathrm{cmH}_{2} \mathrm{O}\right)$ & $174.4 \pm 23.2$ & $255.5 \pm 23.8$ & $81.1^{*}$ & $<0.001$ & 69.6 & 123.4 \\
\hline $\operatorname{MEP}\left(\mathrm{cmH}_{2} \mathrm{O}\right)$ & $156.7 \pm 31.4$ & $219.6 \pm 26.4$ & $62.9 *$ & $<0.001$ & 49.4 & 97.5 \\
\hline SMIP (ptu) & $1001.2 \pm 389.2$ & $1496.2 \pm 426.7$ & $496.1 *$ & 0.022 & 16.7 & 964.0 \\
\hline SMIP (joules) & $12.6 \pm 3.2$ & $18.7 \pm 3.5$ & $6.10^{*}$ & 0.034 & 3.94 & 9.65 \\
\hline $\mathrm{T}_{\text {cont }}(\operatorname{secs})$ & $16.01 \pm 1.89$ & $21.40 \pm 1.90$ & $5.39 *$ & $<0.001$ & 3.07 & 6.59 \\
\hline$\Sigma \mathrm{SMIP}(\mathrm{ptu})$ & $38423.0 \pm 8702$ & $49558.3 \pm 9361$ & $11135.3^{*}$ & 0.026 & 1435.4 & 20819.2 \\
\hline $\mathrm{T}_{\lim }(\min )$ & $3.42 \pm 1.71$ & $4.02 \pm 1.62$ & $0.60 *$ & 0.008 & 0.18 & 1.00 \\
\hline \multicolumn{7}{|l|}{ Pulmonary Function } \\
\hline $\mathrm{FVC}(\mathrm{L})$ & $5.32 \pm 0.96$ & $5.94 \pm 1.1$ & $0.62 *$ & 0.004 & 0.26 & 0.83 \\
\hline $\mathrm{FEV}_{1}(\mathrm{~L})$ & $4.87 \pm 0.84$ & $5.52 \pm 0.97$ & $0.65^{*}$ & $<0.001$ & 0.43 & 0.79 \\
\hline $\mathrm{FIV}_{1}(\mathrm{~L})$ & $4.22 \pm 0.75$ & $4.53 \pm 0.62$ & $0.31 *$ & 0.024 & 0.21 & 0.43 \\
\hline $\mathrm{RV}(\mathrm{L})$ & $1.41 \pm 0.3$ & $1.35 \pm 0.4$ & -0.06 & 0.461 & 0.11 & -0.21 \\
\hline TLC (L) & $6.73 \pm 1.4$ & $7.23 \pm 1.5$ & $0.50 *$ & $<0.001$ & 0.43 & 0.71 \\
\hline $\mathrm{DL}_{\mathrm{CO}}\left(\mathrm{mL} \cdot \mathrm{min}^{-1} \cdot \mathrm{mmHg}^{-1}\right)$ & $35.23 \pm 6.4$ & $39.83 \pm 7.1$ & $4.60 *$ & 0.009 & 1.21 & 9.69 \\
\hline $\mathrm{K}_{\mathrm{CO}}\left(\mathrm{mL}^{\mathrm{min}}{ }^{-1} \mathrm{mmHg}^{-1}\right.$ of $\left.\mathrm{V}_{\mathrm{A}}^{-1}\right)$ & $4.76 \pm 0.8$ & $4.89 \pm 0.9$ & 0.13 & 0.830 & -0.12 & 0.98 \\
\hline
\end{tabular}

Values are means \pm SD. MIP, maximal inspiratory pressure; MEP, maximal expiratory pressure; SMIP, sustained maximal inspiratory pressure (pressure time unit and joules); $\mathrm{T}_{\text {cont }}$, inspiratory time of contraction; $\Sigma \mathrm{SMIP}$, the total area of SMIPs performed to the point of failure summed; $\mathrm{T}_{\text {lim }}$, time to fatigue (performance test); FVC; forced vital capacity; $\mathrm{FEV}_{1}$, forced expiratory volume in $1 \mathrm{sec} ; \mathrm{FIV}_{1}$, forced inspiratory volume in $1 \mathrm{sec} ; \mathrm{RV}$, residual lung volume; TLC, total lung capacity; DL $\mathrm{CO}$, diffusion capacity of the lung for carbon monoxide; $\mathrm{K}_{\mathrm{CO}}$, transfer coefficient $\left(\mathrm{DL}_{\mathrm{CO}} / \mathrm{V}_{\mathrm{A}}\right)$; CI, confidence interval. $*$ - significant difference $(P<0.05)$. 
Table 2. Respiratory muscle function and pulmonary function values for swim training (ST) and sham- inspiratory muscle training (SHAM-IMT) group

\begin{tabular}{|c|c|c|c|c|c|c|}
\hline & Pre & Post & Diff & $P$-value & Lower $95 \%$ CI & Upper $95 \%$ CI \\
\hline \multicolumn{7}{|l|}{ Respiratory Muscle Function } \\
\hline $\mathrm{MIP}\left(\mathrm{cmH}_{2} \mathrm{O}\right)$ & $181.6 \pm 27.3$ & $245.9 \pm 31.8$ & $64.3^{*}$ & 0.008 & 36.7 & 78.4 \\
\hline $\operatorname{MEP}\left(\mathrm{cmH}_{2} 0\right)$ & $163.4 \pm 37.3$ & $227.6 \pm 35.4$ & $64.2^{*}$ & $<0.001$ & 43.2 & 87.8 \\
\hline SMIP (ptu) & $997.4 \pm 298.6$ & $1357.1 \pm 467.9$ & $359.7 *$ & 0.029 & 45.9 & 784.7 \\
\hline SMIP (joules) & $11.9 \pm 2.8$ & $17.6 \pm 3.1$ & $5.70^{*}$ & 0.028 & 2.94 & 8.78 \\
\hline $\mathrm{T}_{\text {cont }}$ (secs) & $15.4 \pm 1.95$ & $20.03 \pm 1.90$ & $4.63 *$ & 0.003 & 1.34 & 6.98 \\
\hline$\Sigma$ SMIP (ptu) & $38675.8 \pm 9845$ & $47674.3 \pm 10096$ & $8998.5^{*}$ & 0.032 & 2765.7 & 16436.8 \\
\hline $\mathrm{T}_{\lim }(\min )$ & $3.54 \pm 1.56$ & $3.92 \pm 1.87$ & $0.38^{*}$ & 0.013 & 0.11 & 1.12 \\
\hline \multicolumn{7}{|l|}{ Pulmonary Function } \\
\hline FVC (L) & $5.21 \pm 0.87$ & $5.78 \pm 1.0$ & $0.57 *$ & 0.003 & 0.18 & 0.87 \\
\hline $\mathrm{FEV}_{1}(\mathrm{~L})$ & $4.96 \pm 0.88$ & $5.59 \pm 0.93$ & $0.63 *$ & $<0.001$ & 0.41 & 0.69 \\
\hline $\mathrm{FIV}_{1}(\mathrm{~L})$ & $4.17 \pm 0.54$ & $4.44+0.69$ & $0.27 *$ & 0.019 & 0.16 & 0.48 \\
\hline $\mathrm{RV}(\mathrm{L})$ & $1.46 \pm 0.3$ & $1.39 \pm 0.3$ & -0.07 & 0.642 & 0.09 & -0.23 \\
\hline TLC (L) & $6.67 \pm 1.7$ & $7.11 \pm 1.9$ & $0.44 *$ & 0.004 & 0.39 & 0.85 \\
\hline $\mathrm{DL}_{\mathrm{CO}}\left(\mathrm{mL}^{\prime} \mathrm{min}^{-1} \mathrm{mmHg}^{-1}\right)$ & $34.79 \pm 7.4$ & $38.69 \pm 7.9$ & $3.90 *$ & 0.019 & 1.07 & 9.34 \\
\hline $\mathrm{K}_{\mathrm{CO}}\left(\mathrm{mL} \min ^{-1} \mathrm{mmHg}^{-1}\right.$ of $\left.\mathrm{V}_{\mathrm{A}}^{-1}\right)$ & $4.67 \pm 0.9$ & $4.77 \pm 0.9$ & 0.10 & 0.812 & -0.15 & 1.12 \\
\hline
\end{tabular}

Values are means \pm SD. MIP, maximal inspiratory pressure; MEP, maximal expiratory pressure; SMIP, sustained maximal inspiratory pressure (pressure time unit and joules); $\mathrm{T}_{\text {cont, }}$, inspiratory time of contraction; $\Sigma$ SMIP, the total area of SMIPs performed to the point of failure summed; $\mathrm{T}_{\text {lim }}$, time to fatigue (performance test); FVC; forced vital capacity; $\mathrm{FEV}_{1}$, forced expiratory volume in $1 \mathrm{sec} ; \mathrm{FIV}_{1}$, forced inspiratory volume in $1 \mathrm{sec} ; \mathrm{RV}$, residual lung volume; TLC, total lung capacity; DL $\mathrm{CO}$, diffusion capacity of the lung for carbon monoxide; $\mathrm{K}_{\mathrm{CO}}$, transfer coefficient $\left(\mathrm{DL}_{\mathrm{CO}} / \mathrm{V}_{\mathrm{A}}\right) ; \mathrm{CI}$, confidence interval. * - significant difference $(P<0.05)$. 
Table 3. Respiratory muscle function and pulmonary function values for swim training (ST) group (no inspiratory muscle training)

\begin{tabular}{|c|c|c|c|c|c|c|}
\hline & Pre & Post & Diff & $P$-value & Lower $95 \%$ CI & Upper $95 \% \mathrm{Cl}$ \\
\hline \multicolumn{7}{|l|}{ Respiratory Muscle Function } \\
\hline $\operatorname{MIP}\left(\mathrm{cmH}_{2} \mathrm{O}\right)$ & $184.6 \pm 33.2$ & $242.2 \pm 24.2$ & $57.6^{*}$ & 0.012 & 23.4 & 72.3 \\
\hline $\operatorname{MEP}\left(\mathrm{cmH}_{2} \mathrm{O}\right)$ & $152.7 \pm 29.6$ & $221.5 \pm 32.4$ & $68.8^{*}$ & $<0.001$ & 38.7 & 84.3 \\
\hline SMIP (ptu) & $898.6 \pm 296.2$ & $1236.8 \pm 351.2$ & $338.2 *$ & 0.032 & 162.7 & 562.6 \\
\hline SMIP (joules) & $12.3 \pm 2.4$ & $18.1 \pm 3.8$ & $5.8^{*}$ & 0.039 & 2.67 & 8.89 \\
\hline $\mathrm{T}_{\text {cont }}(\mathrm{secs})$ & $15.7 \pm 1.67$ & $20.96 \pm 1.62$ & $5.26^{*}$ & 0.009 & 1.12 & 8.69 \\
\hline$\Sigma \mathrm{SMIP}(\mathrm{ptu})$ & $38967.6 \pm 10211$ & $48271.4 \pm 10376$ & $9303.8 *$ & 0.016 & 3104.4 & 18643.6 \\
\hline $\mathrm{T}_{\lim }(\min )$ & $3.34 \pm 1.94$ & $3.81 \pm 1.63$ & $0.47^{*}$ & 0.009 & 0.16 & 1.06 \\
\hline \multicolumn{7}{|l|}{ Pulmonary Function } \\
\hline $\mathrm{FVC}(\mathrm{L})$ & $5.27 \pm 0.90$ & $5.86 \pm 1.1$ & $0.59 *$ & 0.005 & 0.21 & 0.78 \\
\hline $\mathrm{FEV}_{1}(\mathrm{~L})$ & $4.94 \pm 0.76$ & $5.49 \pm 0.84$ & $0.55^{*}$ & $<0.001$ & 0.46 & 0.73 \\
\hline $\operatorname{FIV}_{1}(\mathrm{~L})$ & $4.26 \pm 0.62$ & $4.58 \pm 0.59$ & $0.32 *$ & 0.034 & 0.19 & 0.52 \\
\hline $\mathrm{RV}(\mathrm{L})$ & $1.43 \pm 0.3$ & $1.40 \pm 0.3$ & -0.03 & 0.534 & 0.08 & -0.18 \\
\hline $\mathrm{TLC}(\mathrm{L})$ & $6.70 \pm 1.3$ & $7.13 \pm 1.2$ & $0.43 *$ & 0.014 & 0.34 & 0.78 \\
\hline $\mathrm{DL}_{\mathrm{CO}}\left(\mathrm{mL} \cdot \mathrm{min}-1 . \mathrm{mmHg}^{-1}\right)$ & $34.76 \pm 6.1$ & $37.85 \pm 6.5$ & $3.09 *$ & 0.026 & 0.11 & 7.09 \\
\hline $\mathrm{K}_{\mathrm{CO}}\left(\mathrm{mL} \cdot \min ^{-1} \mathrm{mmHg}^{-1}\right.$ of $\left.\mathrm{VA}^{-1}\right)$ & $4.69 \pm 0.7$ & $4.72 \pm 0.8$ & 0.03 & 0.921 & -0.56 & 1.36 \\
\hline
\end{tabular}

Values are means \pm SD. MIP, maximal inspiratory pressure; MEP, maximal expiratory pressure; SMIP, sustained maximal inspiratory pressure (pressure time unit and joules); $\mathrm{T}_{\text {cont }}$, inspiratory time of contraction; $\Sigma$ SMIP, the total area of SMIPs performed to the point of failure summed; $\mathrm{T}_{\mathrm{lim}}$, time to fatigue (performance test); FVC; forced vital capacity; $\mathrm{FEV}_{1}$, forced expiratory volume in $1 \mathrm{sec} ; \mathrm{FIV}_{1}$, forced inspiratory volume in $1 \mathrm{sec} ; \mathrm{RV}$, residual lung volume; TLC, total lung capacity; DL $\mathrm{CO}$, diffusion capacity of the lung for carbon monoxide; $\mathrm{K}_{\mathrm{CO}}$, transfer coefficient $\left(\mathrm{DL}_{\mathrm{CO}} / \mathrm{V}_{\mathrm{A}}\right) ; \mathrm{CI}$, confidence interval. * - significant difference $(P<0.05)$. 


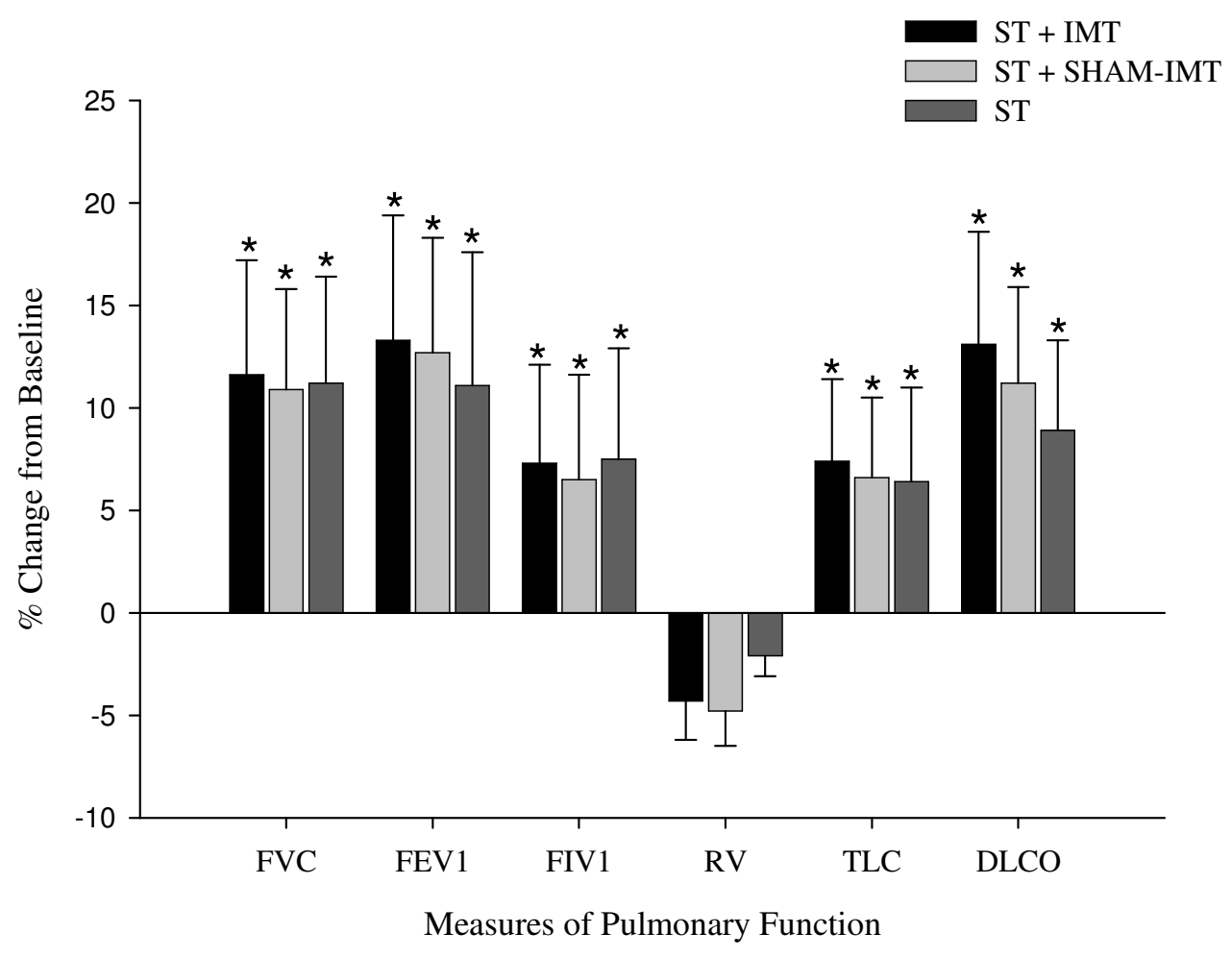

Figure 1a.

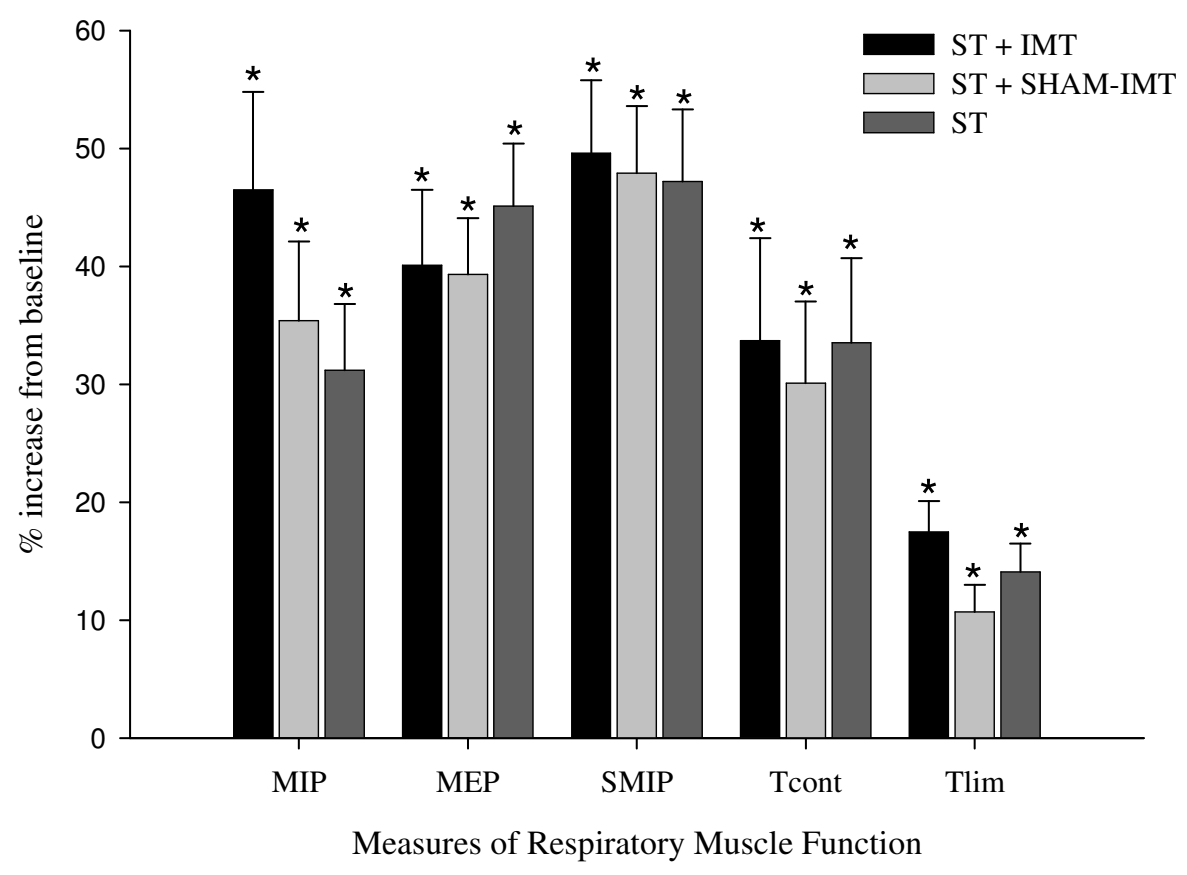

Figure 1b. 


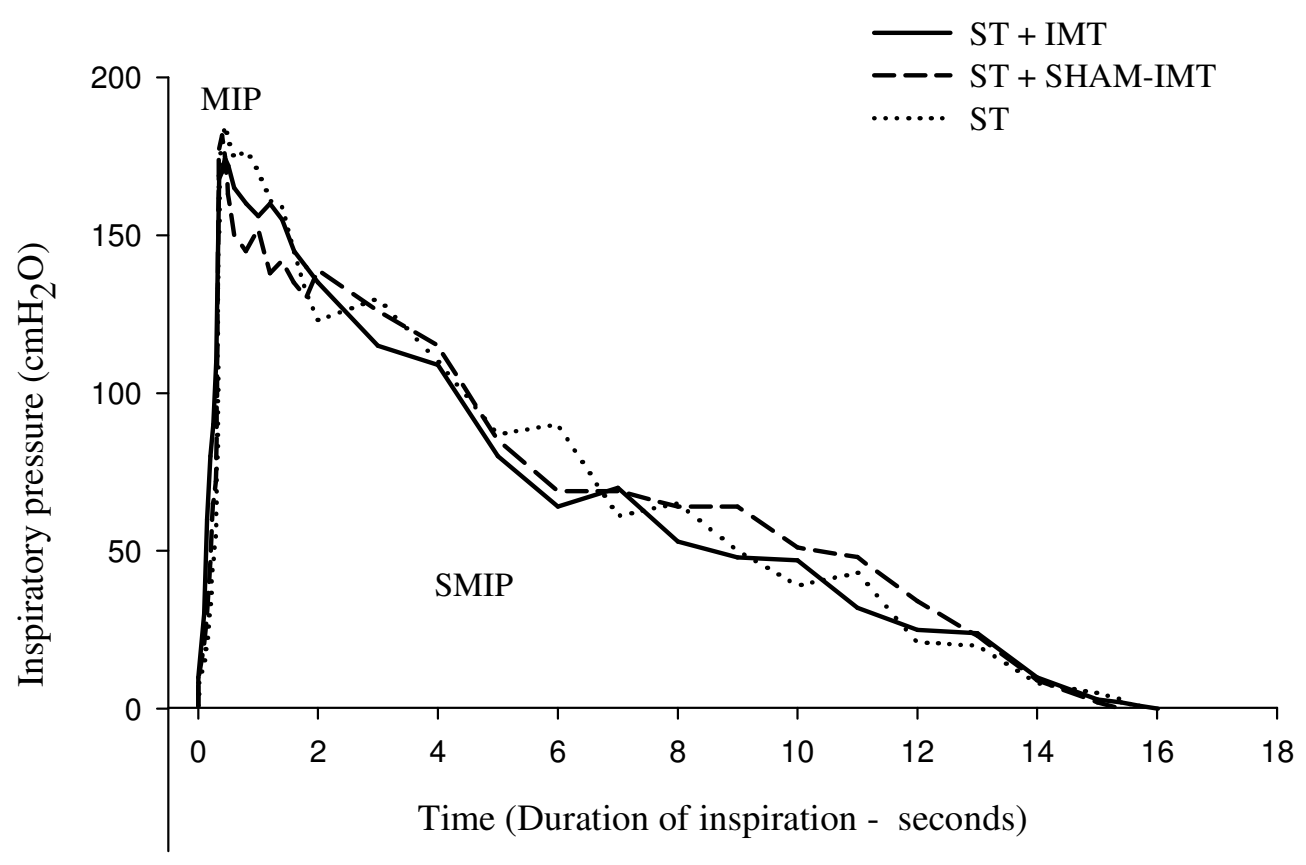

Figure 2a.

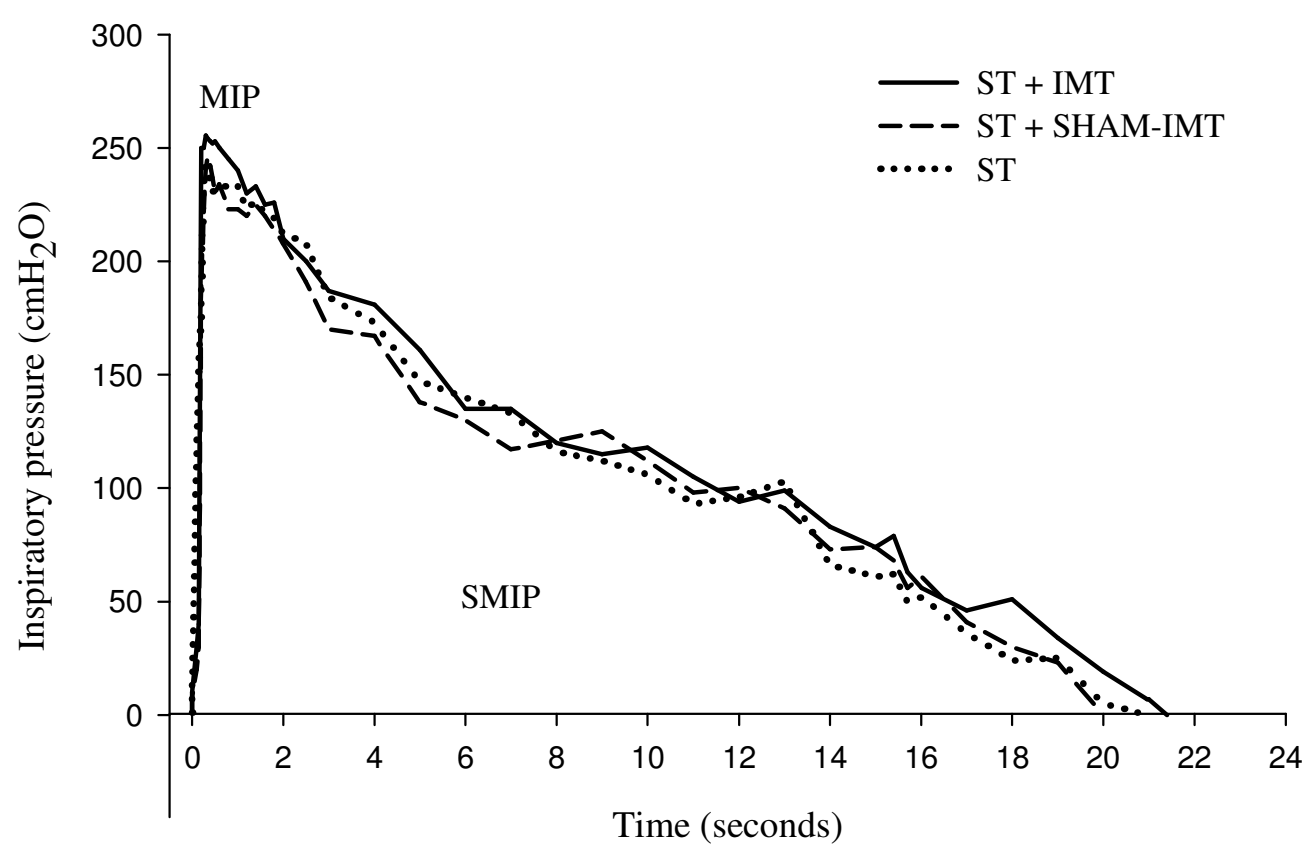

Figure $2 b$. 
